\title{
Searching for Evidence-Based Medicine in the Literature Part 1: The Start
}

\author{
Barbara A. Bartkowiak, MLIS, MST, George E. Magnin Medical Library, Marshfield Clinic, Marshfield, Wisconsin
}

\section{REPRINT REQUESTS:}

Barbara A. Bartkowiak, MLIS, MST

George E. Magnin Medical Library

Marshfield Clinic

1000 N. Oak Avenue

Marshfield, WI 54449

Tel: $715-389-4285$

Fax: 715-389-5366

Email: bartkowiak.barbara@mcrf.mfldclin.edu

\section{KEYWORDS:}

Evidence-based medicine; Computer-assisted instruction; Internet

RECEIVED:

OCTOBER 7, 2004

ACCEPTED:

OCTOBER 27, 2004

Clinical Medicine \& Research

Volume 2, Number 4: 254-255

(02004 Clinical Medicine \& Research

http://www.mfldclin.edu/clinmedres
Evidence-based medicine (EBM) permeates much of current clinical discussion, yet it remains difficult to use in daily practice. Part of that difficulty arises from its definition. EBM is not just searching the literature for good information, rather it is a much more rigorous methodology in gleaning substantive information about patient conditions and in analyzing the strength of those results. EBM is a bit disparate in its nature; there is usually no one-stop shopping for finding it. Another obstacle to its implementation is the level of searching sophistication that EBM requires. There are many web sites, books, and articles about EBM and its nuances, but few make it simple to use. This article is the first of a 3-part series in locating EBM resources that make it easier to identify, perform and appraise relevant clinical information.

Since one of the first tenets of EBM requires well-defined questions about patient care to begin the search, it's important to identify and structure the query appropriately. Several web sites refer to questions as either background or foreground types. While a background question focuses on general factors of a condition, the foreground question specifies a particular patient context. The former question could be answered in a medical textbook whereas the foreground query may require a search of more detailed journal literature.

One of the best web sites to assist the practitioner in the formation of a well constructed foreground question is "The Well-Built Clinical Question" from the Health Services Library of the University of Carolina (http://www.hsl.unc.edu/Services/Tutorials/EBM/Question.htm). This section of the web site sets up a case study and walks the reader through the steps involved to formulate and differentiate the essential parts of a query. It is a part of an extended introduction to EBM that separates the tutorial into manageable, time-friendly units. The user may proceed easily to the next section, delve into more detail with supplemental information or exit. With instant and eye appealing feedback, the learner also can test his/her new knowledge for each question relating to the material covered. Visually, the web site uses strong contrast, color and a clear font size to persuade the eye to move on, and the provision of a glossary and references grounds the content. 
The University of Manchester's "Evidence 4U" web site (http://www.evidence4u.man.ac.uk/modules) offers a briefer pathway to developing a well-constructed question. While the general concept of query structure may seem obvious, in EBM it is a critical component. This web site uses a chart to differentiate those components, part of a more focused search preparation. The PICO structure (an acronym for patient or population, intervention or exposure, comparisons, if needed, and outcomes) is graphically charted into sections with questions that prompt the reader toward a richer result. To promote the pathway, the web site offers forms that can be used to focus the components of a question. Brief and cogent descriptions of the types of study designs further sharpen the clarity of the question formation. Occasionally one might get distracted by the multiple options of forms, further discussion choices, or alternative concepts, but this gives the user an opportunity to either "dip or dive" into the material. The only drawback to this site is that one needs to register and login using a password, but it is a free, reliable and authoritative source.

All of this preparation in the construction of a question can be a solid investment of time and increases efficiency of the search. New users often plug their questions into the search box as strings of words without regard to language inconsistencies in the forms of synonyms, singular versus plural, or variant spellings. Database searching demands clearly focused questions with concepts that are entered separately and then combined later. The time that may be required for setting up the question is worth the effort in the next stage of the EBM methodology - resource selection and search execution. Performing the search will be discussed in the 2nd of this 3-part series on evidence-based medicine resources that will appear in the February 2005 issue of Clinical Medicine \& Research. 